\title{
Aktuelle Übersicht zur Therapieprotokollen der ADO beim malignen Melanom
}

J. Ulrich ${ }^{1}$

P. Mohr ${ }^{2}$

A. Hauschild ${ }^{3}$

\section{Up-to-Date Overview on Treatment Protocols of the ADO in Malignant \\ Melanoma}

Seit ihrer Gründung im Jahre 1991 hat die Arbeitsgemeinschaft Dermatologische Onkologie (ADO) als eines ihrer Hauptziele die Planung und Durchführung qualitativ hochwertiger klinischer und experimentell-klinischer Studien mit dem Ziel der Therapieoptimierung angesehen. Bis dato waren dermato-onkologische Studien in Deutschland in der Regel als kleinere monozentrische Studien durchgeführt worden. Durch die Etablierung fallzahlstarker multizentrischer Studien, die vor allem als Therapieoptimierungsprotokolle (TOP) geplant wurden, sollte eine Qualität der Studien erreicht werden, die sowohl im „internen“ Vergleich der Arbeitsgemeinschaften der Deutschen Krebsgesellschaft als auch im externen Vergleich mit anderen Organisationen (wie z.B. EORTC oder ECOG) standhalten. Durch die breite Akzeptanz der Protokolle in der Dermatologie konnte die Qualität der Versorgung dermato-onkologischer Patienten im letzten Jahrzehnt entscheidend verbessert werden.

Das erste randomisierte Therapieoptimierungsprotokoll (TOP) der ADO für das Stadium IV des malignen Melanoms wurde 1994 initiiert und bis zum April 1998 abgeschlossen. Die Ergebnisse der Studie wurden mittlerweile publiziert [Hauschild A et al. (2001) Br J Cancer 84:1036 - 1042]. In den Jahren 1997 und 1998 wurde das Spektrum der TOP um Protokolle für die adjuvante Therapiesituation bereichert. Eine Aufstellung über die bislang abgeschlossenen bzw. nicht mehr rekrutierenden TOP zeigt Tab. 1.

Mittlerweile konnte die Studienlandschaft so strukturiert werden, dass im Prinzip für alle klinischen Stadien des malignen Melanoms sowie für die adjuvante als auch die palliative Therapiesituation entsprechende Behandlungsprotokolle zur Verfügung stehen. Trotz gewisser Therapiestandards für die adjuvante und die palliative Therapiesituation präferiert die ADO den Einschluss von Melanompatienten in kontrollierte Therapieprotokolle, um die nach wie vor unbefriedigende Therapiesituation systematisch zu verbessern. Eine Übersicht über die derzeit laufenden Protokolle für das maligne Melanom gibt Tab. 2. Detailinformationen für die einzelnen Protokolle befinden sich auf der Homepage der ADO (www.ado-homepage.de). Hier können die Protokollsynopsis eingesehen und Kontakt mit den Leitern der Protokolle aufgenommen werden.
Das 12. Gesetz zur Änderung des Arzneimittelgesetzes vom 30.7.2004, das am 6. 8.2004 in Kraft getreten ist, bringt im Hinblick auf klinische Prüfungen von Arzneimitteln eine Vielzahl von Neuerungen und wird somit nachhaltig die Studienlandschaft beeinflussen. Unter das Gesetz fallen alle klinischen Prüfungen, die nach der Bekanntmachung des Gesetzestextes am 6. 8. 2004 bei einer Ethikkommission eingereicht wurden und werden. Somit sind die in Tab. 2 aufgeführten Therapieprotokolle von den neuen Regelungen ausgenommen und können nach den bislang gültigen Normen durchgeführt werden.

Bislang haben über 90 überwiegend dermatologische Zentren in Deutschland, Östereich und der Schweiz mehr als 3200 Patienten im Rahmen von Therapieprotokollen der ADO behandelt. Für eine vergleichsweise "kleine“ Studiengruppe wie die ADO ein bemerkenswertes Ergebnis, auf das die ADO und ihre Mitglieder mit Recht stolz sein können. Auf diesem Wege möchte der amtierende Vorstand der ADO allen Kolleginnen und Kollegen, die zu diesem Ergebnis beigetragen haben, recht herzlich für die gute Zusammenarbeit danken.

${ }^{1}$ Klinik für Dermatologie und Venerologie, Otto-von-Guericke-Universität Magdeburg

2 Dermatologisches Zentrum, Elbeklinikum Buxtehude

${ }^{3}$ Klinik für Dermatologie, Venerologie und Allergologie, Universitätsklinikum Schleswig-Holstein,

Campus Kiel

Priv.-Doz. Dr. med. Jens Ulrich · Klinik für Dermatologie und Venerologie · Otto-von-Guericke-

Universität Magdeburg · Leipziger Straße 44 · 39120 Magdeburg ·

E-mail: jens.ulrich@medizin.uni-magdeburg.de

Bibliografie

Akt Dermatol 2004; 30: 456-458 @ Georg Thieme Verlag Stuttgart · New York .

DOI 10.1055/s-2004-826029 · ISSN 0340-2541 
Tab. 1 Übersicht zu ADO-Protokollen beim malignen Melanom mit abgeschlossener Rekrutierung

\begin{tabular}{|c|c|c|c|c|c|c|}
\hline \multirow{2}{*}{$\begin{array}{l}\text { Studien-Nr. } \\
\text { PAL-1 }\end{array}$} & \multirow{2}{*}{$\begin{array}{l}\text { Therapieschema } \\
\text { a) DTIC + IFN- } \alpha\end{array}$} & \multirow{2}{*}{$\begin{array}{l}\text { Pat.-zahl } \\
\\
300\end{array}$} & \multicolumn{2}{|c|}{$\begin{array}{l}\text { Patientenrekrutierung } \\
\text { Beginn } \quad \text { Ende }\end{array}$} & \multirow{2}{*}{$\begin{array}{l}\text { Aktueller Status/(Zwischen)-ergebnis } \\
\text { Ergebnisse publiziert*/Kein Unterschied } \\
\text { für Überleben- und Remissionsrate }\end{array}$} & \multirow{2}{*}{$\begin{array}{l}\text { LKP } \\
\text { Prof. Tilgen (Homburg) }\end{array}$} \\
\hline & & & 1994 & 1998 & & \\
\hline & b) DTIC + IFN- $\alpha+$ IL-2 & & & & & \\
\hline \multirow[t]{2}{*}{ PAL-2 } & a) Temozolomid & 300 & 1998 & 2002 & Publikation in Vorbereitung & Prof. Kaufmann (Frankfurt) \\
\hline & b) Temozolomid + IFN- $\alpha$ & & & & & \\
\hline $\begin{array}{l}\text { PAL-2 } \\
\text { (Amendment) }\end{array}$ & $\begin{array}{l}\text { b) Temozolomid + } \\
\text { PEG-IFN- } \alpha 2 \text { b }\end{array}$ & & & & & \\
\hline \multirow[t]{2}{*}{ PAL-3 } & a) Paclitaxel + Carboplatin & 37 & 2000 & 2002 & $\begin{array}{l}\text { Ergebnisse publiziert**/Kein sign. } \\
\text { Unterschied }\end{array}$ & $\begin{array}{l}\text { Prof. Schadendorf } \\
\text { (Mannheim) }\end{array}$ \\
\hline & b) Paclitaxel & & & & & \\
\hline PAL-4 & a) DTIC & 109 & 2000 & 2003 & Publikation in Vorbereitung & $\begin{array}{l}\text { Prof. Schadendorf } \\
\text { (Mannheim) }\end{array}$ \\
\hline PAL-6 & $\begin{array}{l}\text { Selektive Chemotherapie } \\
\text { nach Chemo-Sensitivitäts- } \\
\text { testung }\end{array}$ & 95 & 2001 & 2004 & Endauswertung erfolgt & $\begin{array}{l}\text { Prof. Reinhold (Homburg/ } \\
\text { Bonn) }\end{array}$ \\
\hline \multirow[t]{3}{*}{$A D J-1$} & a) Beobachtung & 444 & 1997 & 2001 & $\begin{array}{l}\text { Manuskript eingereicht/DFS und OS } \\
\text { für Arm B sign. höher als Arm A und } \\
\text { Arm C }\end{array}$ & Prof. Garbe (Tübingen) \\
\hline & b) IFN- $\alpha 2 a$ & & & & & \\
\hline & c) IFN- $\alpha 2 a+D T I C$ & & & & & \\
\hline \multirow[t]{2}{*}{$A D J-2$} & $\begin{array}{l}\text { a) IFN- } \alpha 2 \mathrm{~b} \text { (Mittelhoch- } \\
\text { dosis initial) }\end{array}$ & 697 & 1997 & 2002 & $\begin{array}{l}\text { Publikation in Vorbereitung/Kein sign. } \\
\text { Überlebensvorteil für DFS und OS }\end{array}$ & Prof. Hauschild (Kiel) \\
\hline & b) IFN- $\alpha 2 b$ (Niedrigdosis) & & & & & \\
\hline$A D J-3$ & a) Beobachtung & 142 & 1998 & 2001 & $\begin{array}{l}\text { Endauswertung vorgestellt/kein sig. } \\
\text { Vorteil für DFS und OS }\end{array}$ & Prof. Garbe (Tübingen) \\
\hline
\end{tabular}

LKP = Leiter der klinischen Prüfung nach Arzneimittelgesetz; ADJ = Therapieprotokoll für die adjuvante Situation; PAL = Therapieprotokoll für die palliative Situation; IFN = Interferon; IL = Interleukin; DTIC = Dacarbazin; DFS = Disease-free survival; OS = Overall survival; sign. = signifikant

* Hauschild A et al. Brit J Cancer 2001; 84: 1036-1042

** Zimpfer-Rechner C et al. Melanoma Res 2003; 13: $531-536$ 
Tab. 2 Übersicht zu laufenden ADO-Protokollen beim malignen Melanom

\begin{tabular}{|c|c|c|c|c|c|c|}
\hline \multirow{2}{*}{$\begin{array}{l}\text { Studien-Nr. } \\
\text { PAL-5 }\end{array}$} & \multirow{2}{*}{$\begin{array}{l}\text { Therapieschema } \\
\text { Temozolomid in veränderter } \\
\text { Dosierung bei Hirnmetastasen in a) } \\
\text { chemonaiven und b) vorbehandelten } \\
\text { Patienten }\end{array}$} & \multirow{2}{*}{$\begin{array}{l}\text { Beginn } \\
2001\end{array}$} & \multicolumn{2}{|c|}{$\begin{array}{l}\text { Patientenrekrutierung } \\
\text { Ist } \quad \text { Soll }\end{array}$} & \multirow{2}{*}{$\begin{array}{l}\text { Kommentar } \\
\text { Aufstockung auf } 66 \text { je } \\
\text { Arm bei mehr als } \\
3 \text { Ansprechern möglich }\end{array}$} & \multirow{2}{*}{$\begin{array}{l}\text { LKP } \\
\text { Prof. Schadendorf } \\
\text { (Mannheim) }\end{array}$} \\
\hline & & & 41 & 42 & & \\
\hline \multirow[t]{2}{*}{ PAL-7 } & a) Beobachtung & 2000 & 16 & 167 & - & Prof. Enk (Heidelberg) \\
\hline & $\begin{array}{l}\text { b) IFN- } \alpha+\text { IL-2 Erhaltungstherapie } \\
\text { im Stad. IV }\end{array}$ & & & & & \\
\hline \multirow[t]{2}{*}{ PAL-8 } & a) Standard palliative care & 2001 & 87 & 200 & - & PD Ulrich (Magdeburg) \\
\hline & $\begin{array}{l}\text { b) Standard palliative care + } \\
\text { DVP-Polychemotherapie }\end{array}$ & & & & & \\
\hline \multirow[t]{2}{*}{$A D J-5$} & a) IFN- $\alpha 2 b$ (Hochdosis) & 2004 & 53 & 600 & - & Dr. Mohr (Buxtehude) \\
\hline & b) IFN- $\alpha 2 b$ (Hochdosis, alternierend) & & & & & \\
\hline \multirow[t]{2}{*}{$A D J-6$} & a) PEG- IFN- $\alpha 2 a$ & 2004 & - & 880 & $\begin{array}{l}\text { Beginn im Oktober } 2004 \\
\text { vorgesehen }\end{array}$ & Prof. Garbe (Tübingen) \\
\hline & b) IFN- $\alpha 2 a$ & & & & & \\
\hline \multirow[t]{2}{*}{$A D J-7$} & a) ELND nach WLB & 2004 & - & 438 & $\begin{array}{l}\text { Protokoll in Kooperation } \\
\text { mit der VOD, zur Zeit } \\
\text { Begutachtung bei der } \\
\text { Deutschen Krebshilfe }\end{array}$ & Prof. Stadler (Minden) \\
\hline & b) Beobachtung nach SLNB & & & & & \\
\hline
\end{tabular}

Erklärung der Abkürzungen s. Tab. 1; DVP = Polychemotherapie mit Dacarbazin, Vindesin und Platinex; ELND = elektive radikale Lymphknotendissektion, WLB = Wächter-Lymphknotenbiospie 